\title{
Editorial
}

\section{MultiSensor Processing for Signal Extraction and Applications}

\author{
Chong-Yung Chi, ${ }^{1}$ Ta-Sung Lee, ${ }^{2}$ Zhi-Quan Luo, ${ }^{3}$ Yue Wang, ${ }^{4}$ and Kung Yao ${ }^{5}$ \\ ${ }^{1}$ Institute of Communications Engineering, and Department of Electrical Engineering, National Tsing Hua University, 101, Sec. 2, \\ Kuang Fu Road, Hsinchu, Taiwan 30013 \\ ${ }^{2}$ Department of Communication Engineering, National Chiao Tung University, 1001 Ta Hsueh Road, Hsinchu, Taiwan 300 \\ ${ }^{3}$ Department of Electrical and Computer Engineering, University of Minnesota, 200 Union Street SE, Minneapolis, \\ MN 55455, USA \\ ${ }^{4}$ Computational Bioinformatics and Bioimaging Laboratory, Advanced Research Institute, 4300 Wilson Boulevard, Suite 750, \\ Arlington, VA 22203, USA \\ ${ }^{5}$ Electrical Engineering Department, University of California, Los Angeles, CA 90095, USA
}

Received 29 August 2006; Accepted 29 August 2006

Copyright ( $) 2006$ Chong-Yung Chi et al. This is an open access article distributed under the Creative Commons Attribution License, which permits unrestricted use, distribution, and reproduction in any medium, provided the original work is properly cited.

Source signal extraction from heterogeneous measurements has a wide range of applications in many scientific and technological fields, for example, digital communication, speech and acoustic signal processing, as well as biomedical pattern analysis. In these applications, the use of a multisensor system allows simultaneous reception of multiple signals which, when appropriately processed, can deliver significant performance improvement over a single-sensor system. A key component of any multisensor system is the signal processing module which ideally should maximally exploit the diversity present in the multiple received copies of the mixed source signals. The ultimate goal of multisensor signal processing is to offer robust high quality signal extraction under realistic assumptions with minimal computational complexity. Despite continued progress in the past few decades, multisensor-based signal processing techniques have remained a major research focus of the signal processing community. Currently there are major on-going research efforts in high quality signal extraction, realistic theoretical modeling of real-world problems, algorithm complexity reduction, and efficient real-time implementation. In response to the growing interest from industry, academia, and government agencies in the research and development of multisensor signal processing systems, this special issue is edited so as to provide a snapshot of the state-of-the-art in multisensor signal processing research.

This special issue is composed of four groups of contributions on signal extraction for multiple-input multipleoutput systems (channels) and applications. The first group consists of one paper (by I. Kacha et al.) studying the equalizer design of a multichannel FIR system with emphases on low computational complexity and robustness to channel conditions, and two papers (by C.-H. Peng et al. and by X. Zheng et al., resp.) exhibiting performance gain (in terms of output signal to interference plus noise ratio or bit-error rate or data rate) as well as computational complexity reduction of wireless communication systems (a multirate DS/CDMA system and an OFDM system) by the use of multiple transmit antennas or multiple receive antennas or both.

The second group consists of one paper (by L. Wang et al.) dealing with speech recognition through the use of microphone-array processing and speaker location estimation, and one paper (by Q. Zeng and W. H. Abdulla) dealing with speech enhancement through a combination of a multi-channel crosstalk resistant adaptive noise cancellation algorithm and a spectrum subtraction algorithm.

The third group consists of three papers on blind source separation (BSS). One paper (by Y. Zhang and M. G. Amin) studies blind separation of nonstationary sources based on spatial time-frequency distributions for performance improvement and relaxation of the condition (required by most BSS algorithms) that the number of sensors must be equal to or larger than the number of sources. Two papers (by R. Mukai et al., and by Y. Mori et al., resp.) present BSS algorithms for acoustic sources or speech signals with performance improvement and/or robustness against channel conditions over conventional BSS algorithms, and one of them is a time-domain approach and the other is a frequencydomain approach and both of them involve independent component analysis. 
The fourth group consists of four papers on specific applications using multisensor processing algorithms, one (by H. Belkacemi and S. Marcos) studying space-time adaptive processing for airborne radar (for computational complexity reduction and nonhomogeneity of data samples), one (by Y. Xie et al.) studying multistatic adaptive microwave imaging for early breast cancer detection (to achieve high resolution and interference suppression by Capon beamforming), one (by J. A. Beracoechea et al.) studying the building of immersive audio systems for the reconstruction or rendering of acoustic fields (by adaptive beamforming techniques for source signal estimation and by a joint audio-video method for source localization), and one (by S. Pandya et al.) studying dipole localization and tracking of vibrational dipole sources underwater (for an engineered artificial lateral line system consisting of a sixteen-element array of hot-wire flow sensors).

We would like to thank the authors of this special issue for their valuable contributions and anonymous reviewers for their significant efforts during the three-round review process. Hopefully, this special issue can serve to advance and stimulate the exciting field of multisensor processing for signal extraction and applications.

Chong-Yung Chi

Ta-Sung Lee Zhi-Quan Luo Yue Wang Kung Yao

Chong-Yung Chi received the Ph.D. degree in electrical engineering from the University of Southern California, in 1983. From 1983 to 1988 , he was with the Jet Propulsion Laboratory, Pasadena, California. He has been a Professor with the Department of Electrical Engineering since 1989 and the Institute of Communications Engineering (ICE) since 1999 (also the Chairman of ICE for 2002-2005), National Tsing Hua University,

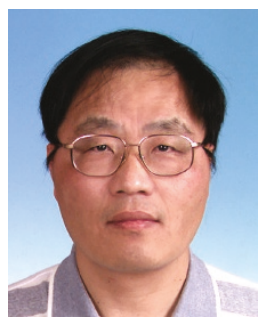

Hsinchu, Taiwan. He coauthored a technical book Blind Equalization and System Identification published by Springer 2006, and published more than 120 technical papers. His current research interests include signal processing for wireless communications, and statistical signal processing. He is a senior member of IEEE. He has been a Technical Program Committee member for many IEEE sponsored workshops, symposiums, and conferences on signal processing and wireless communications, including coorganizer and general cochairman of IEEE SPAWC 2001. He was an Associate Editor of IEEE Trans. Signal Processing (May 2001 through April 2006). Currently, he is an Associate Editor for the IEEE Signal Processing Letters, an Associate Editor for the IEEE Trans. Circuits and Systems II, and a Guest Editor of EURASIP Journal on Applied Signal Processing, a member of Editorial Board of EURASIP Signal Processing Journal, and a member of Technical Committee on Signal Processing Theory and Methods of IEEE Signal Processing Society.
Ta-Sung Lee received the B.S. degree form National Taiwan University, in 1983, the M.S. degree from the University of Wisconsin, Madison, in 1987, and the Ph.D. degree form Purdue University, W. Lafayette, Ind, in 1989, all in electrical engineering. In 1990, he joined the Faculty of National Chiao Tung University (NCTU), Hsinchu, Taiwan, where he now holds a position as Professor and Chairman in the Department of Communication Engineering. His other positions include Technical Advisor at Information and Communications Research Labs of Industrial Technology Research Institute (ITRI), Taiwan, Managing Director of MINDS Research Center, College of EECS, NCTU, and Managing Director of Communications and Computer Training Program, NCTU. He is active in research and development in advanced technologies for wireless communications, such as smart antenna and MIMO technologies, cross-layer system design, and hardware/software prototyping of advanced communication systems, and has published more than 80 original papers. He is recipient of 1999 Young Electrical Engineer Award of the Chinese Institute of Electrical Engineers, and 2001 NCTU Teaching Award.

Zhi-Quan Luo received the B.S. degree in mathematics from Peking University, China, in 1984. During the academic year of 1984 to 1985, he was with Nankai Institute of Mathematics, Tianjin, China. From 1985 to 1989 , he studied at the Department of Electrical Engineering and Computer Science, Massachusetts Institute of Technology, where he received the Ph.D. degree in operations research. In 1989, he joined the

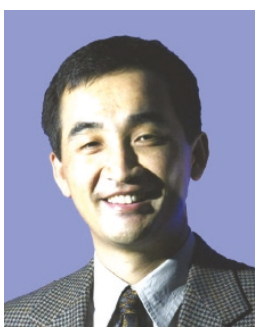

Department of Electrical and Computer Engineering, McMaster University, Hamilton, Canada, where he became a Professor in 1998 and held the Canada Research Chair in Information Processing since 2001. Starting April 2003, he has been a Professor with the Department of Electrical and Computer Engineering and holds an endowed ADC research Chair in Wireless Telecommunications with the Digital Technology Center at the University of Minnesota. His research interests lie in the union of large-scale optimization, information theory and coding, data communications and signal processing. He is a member of SIAM and MPS. He is presently serving as an Associate Editor for several international journals including SIAM Journal on Optimization, Mathematics of Computation, Mathematics of Operations Research, and IEEE Transactions on Signal Processing.

Yue Wang received his B.S. and M.S. degrees in electrical and computer engineering from Shanghai Jiao Tong University, in 1984 and 1987, respectively. He received his Ph.D. degree in electrical engineering from University of Maryland Graduate School in 1995. In 1996, he was a Postdoctoral Fellow at Georgetown University School of Medicine. From 1996 to 2003, he was an Assistant and later Associate Professor of

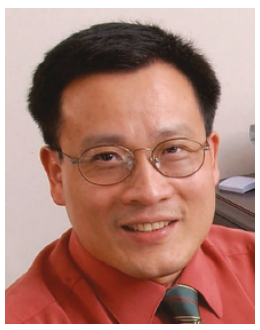
electrical engineering, the Catholic University of America, Washington DC. In 2003, he joined Virginia Polytechnic Institute and State University (Virginia Tech), Arlington, Va, and is currently a Professor of electrical, computer, and biomedical engineering. $\mathrm{He}$ is also an Affiliated Faculty Member of the Johns Hopkins Medical Institutions. He became a Fellow of The American Institute for Medical and Biological Engineering (AIMBE) in 2004. 
His research interests focus on computational intelligence, machine learning, pattern recognition, statistical visualization, and advanced imaging and image analysis, with applications to bioinformatics, computational biology, and biomedical imaging.

Kung Yao received the B.S.E. (highest honors), M.A., and Ph.D. degrees in electrical engineering all from Princeton University, Princeton, NJ. He was an NAS-NRC PostDoctoral Research Fellow at the University of California, Berkeley. Presently, he is a Professor in the Electrical Engineering Department at UCLA. In 1969, he was a Visiting Assistant Professor at MIT. In 19851988, he served as an Assistant Dean of the

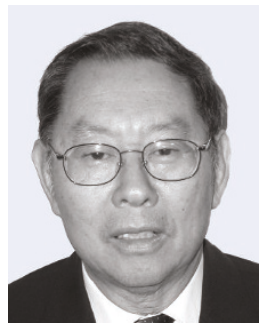
School of Engineering and Applied Science at UCLA. His research interests include sensor array system, digital communication theory, wireless radio system, chaos communications, digital and array processing, systolic and VLSI algorithms, and simulation. He has published over 250 journal and conference papers. He received the IEEE Signal Processing Society's 1993 Senior Award in VLSI Signal Processing. He was the coeditor of a two-volume series of an IEEE Reprint Book on "High Performance VLSI Signal Processing," IEEE Press, 1997. He has served as an Associate Editor for IEEE Transactions on Information Theory, IEEE Transactions on Signal Processing, IEEE Transactions on Circuits and Systems, IEEE Communications Letters, and as a guest editor of numerous special issues. He is a Life Fellow of IEEE. 